\title{
EDITORIAL
}

\section{Thematic reviews}

\author{
Adrian J L Clark ${ }^{\mathbf{1}}$ and David W Ray ${ }^{2}$ \\ ${ }^{1}$ Centre for Endocrinology, William Harvey Research Institute, Barts and The London School of Medicine and Dentistry, London EC1M 6BQ, UK \\ ${ }^{2}$ Endocrine Sciences Research Group, University of Manchester, Manchester M13 9PT, UK \\ (Correspondence should be addressed to A J L Clark; Email: a.j.clark@qmul.ac.uk)
}

The review article has become an increasingly prevalent form of scientific publication over the last decade, and for good reason. In an era of increasing sub-specialization within endocrinology, cross-fertilization of ideas between sub-specialties becomes ever more important. Furthermore, the review provides a readily available means to learn the latest developments and ideas in a given topic, and the Journal has long supported this function by making all its review articles freely available online.

One criticism that might be leveled at our current review article strategy (and that of the majority of other journals dedicated to publishing research in the biological sciences) is that this form of publication strongly reflects the views and achievements of the authors, despite every effort of the peer review process to which all such articles are submitted.
For this reason, we are embarking on a new initiative in this issue to develop a thematic review process. A guest editor will be asked to commission reviews around a central theme from leading authorities in the specified field. These will be accompanied by a short overview from the guest editor outlining the topic and the aims of the collection. In this issue, Domenico Salvatore introduces some of the recent developments and controversies in the deiodinase enzymes with three articles. Other thematic reviews will follow in future issues, but the Journal is open for suggestions from the endocrine community for other topics that would merit this type of analysis. We hope that this initiative is valuable.

Received in final form 3 March 2011

Accepted 7 March 2011 